\title{
ACOMETIMENTO ÓSSEO NA PARACOCCIDIOIDOMICOSE CRÔNICA DISSEMINADA. RELATO DOS PRIMEIROS CASOS NO RIO GRANDE DO SUL
}

\author{
Luiz Carlos Severo, Aventino Alfredo Agostini e \\ Alberto Thomaz Londero
}

\begin{abstract}
São relatados os primeiros casos de paracoccidioidomicose com envolvimento ósseo observados no Rio Grande do Sul. Comentam-se os achados clinico-radiológicos e destacam-se peculiaridades obsentadas nos casos sul-riograndenses.
\end{abstract}

Palauras-chaves: Paracoccidioidomicose. Paracoccidioides brasiliensis. Osso. Articulação.

Paracoccidioidomicose (PCM) é a micose sistêmica mais freqüente no Rio Grande do Sul. Desde os primeiros relatos de casos, em 1942 , vem sendo apontadas algumas peculiaridades das manifestações da micose em nativos residentes no estado: 1) não há registro de caso da forma disseminada subaguda ("juvenil"); 2) nos dois casos da micose observados em crianças as lesões eram circunscritas aos pulmões; 3) o acometimento pulmonar em pacientes com a forma disseminada crônica (tipo "adulto") ocorre em 97 a 100\% dos doentes, em contraposição ao baixo percentual de envolvimento dos linfonodos e da pele; 4) tem sido alta a freqüência de lesões circunscritas aos pulmões em adultos - forma pulmonar crônica. Além disso, em nativos do estado, foram observados 1) o complexo primário pulmonar, em vida e 2) foram comprovadas lesōes pulmonares espontaneamente curadas - lesões residuais ${ }^{3+9}$.

Também, é interessante não ter sido relatado caso de envolvimento ósseo em pacientes sulriograndenses. Esse fato justifica a apresentação de cinco casos de PCM em que houve acometimento ósseo.

\section{MATERIAL E MÉTODOS}

Foram revistos os protocolos clínicos de 505 pacientes com paracoccidioidomicose, diagnosticados no período 1966-1994.

Departamento de Medicina Interna, Universidade Federal do Rio Grande do Sul, Porto Alegre, RS.

Endereço para correspondência: Dr. Luiz Carlos Severo, IPD/Santa Casa. R.Annes Dias 285, 90020-090 Porto Alegre, RS. Fax: (051) 2274040

Recebido para publicacão em 28/11/95.

\section{RESULTADOS}

O acometimento ósseo foi observado em $5 / 505$ dos doentes ( $1 \%$ ); todos eram homens, brancos, com idades entre 31 e 47 anos. Os sintomas, as lesões radiológicas e o diagnóstico constam na Tabela 1. Aspectos radiológicos de lesões osteolíticas em costela, osso frontal e osso coxal são ilustrados nas Figuras 1, 3 e 4, respectivamente. As lesôes óseas podem ocasionar alteraçôes no exame físico. No caso três houve lesão cutânea (Figura 2) e no caso 4 a deformação da cabeça do fêmur levou ao encurtamento do membro inferior esquerdo.

\section{DISCUSSÃO}

O primeiro caso de envolvimento ósseo na PCM foi relatado em 1911 por Pereira e Vianna $^{8}$. Revisões sobre o assunto foram feitas por Silva ${ }^{10}$ e por Marchiori ${ }^{6}$, coligindo, respectivamente, 94 e 92 casos da literatura brasileira e acrescentando 15 e 20 casos pessoais. É de presumir-se que o acometimento ósseo na PCM deva ser mais freqüente, haja visto que em casuísticas de áreas de alta endemicidade da micose o envolvimento do esqueleto foi observado em 3,5 a $16,6 \%$ dos doentes ${ }^{25} 1012$, não tendo sido ultrapassada essa última cifra, mesmo quando feito o estudo sistemático do esqueleto de toda uma série de doentes? .

O acometimento ósseo é mais freqüente em indivíduos jovens, com menos de 20 anos $(27 \text { a } 50 \%)^{6} 10$ e, em certas regiões do Brasil, maior na primeira década da vida ${ }^{6}$. Em crianças, atinge de 10 a $30 \%$ dos pacientes ${ }^{11}$, nos quais, com relativa freqüência, a sintomatologia devido ao acometimento ósseo 
Severo LC, Agostini AA, Londero AT. Acometimento ósseo na paracoccidioidomicose crônica disseminada. Relato dos primeiros casos no Rio Grande do Sul. Revista da Sociedade Brasileira de Medicina Tropical 29:241-244, mai-jun, 1996.

Tabela 1 - Sumário dos sintomas, alterações radiológicas e diagnóstico dos cinco casos.

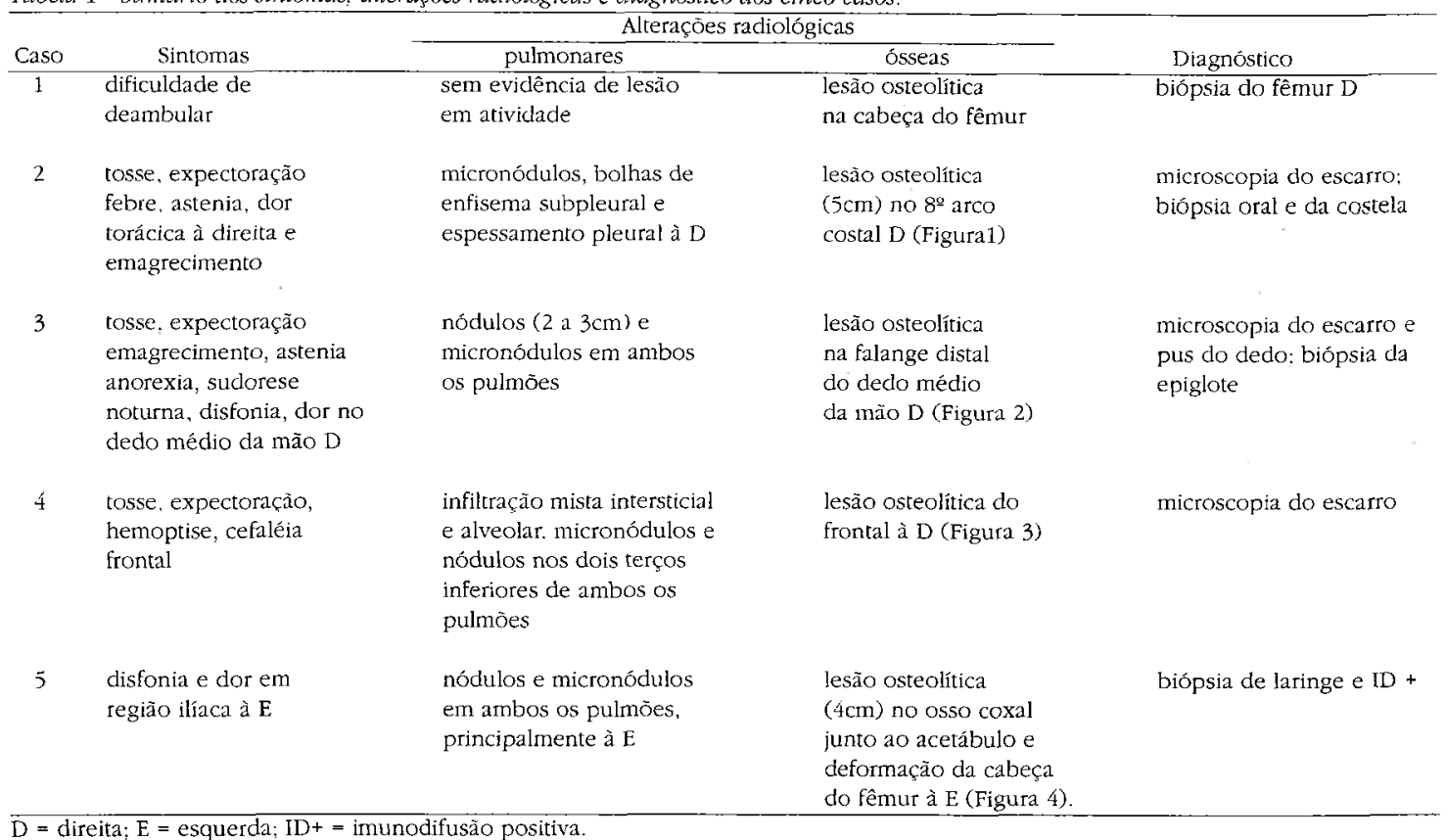
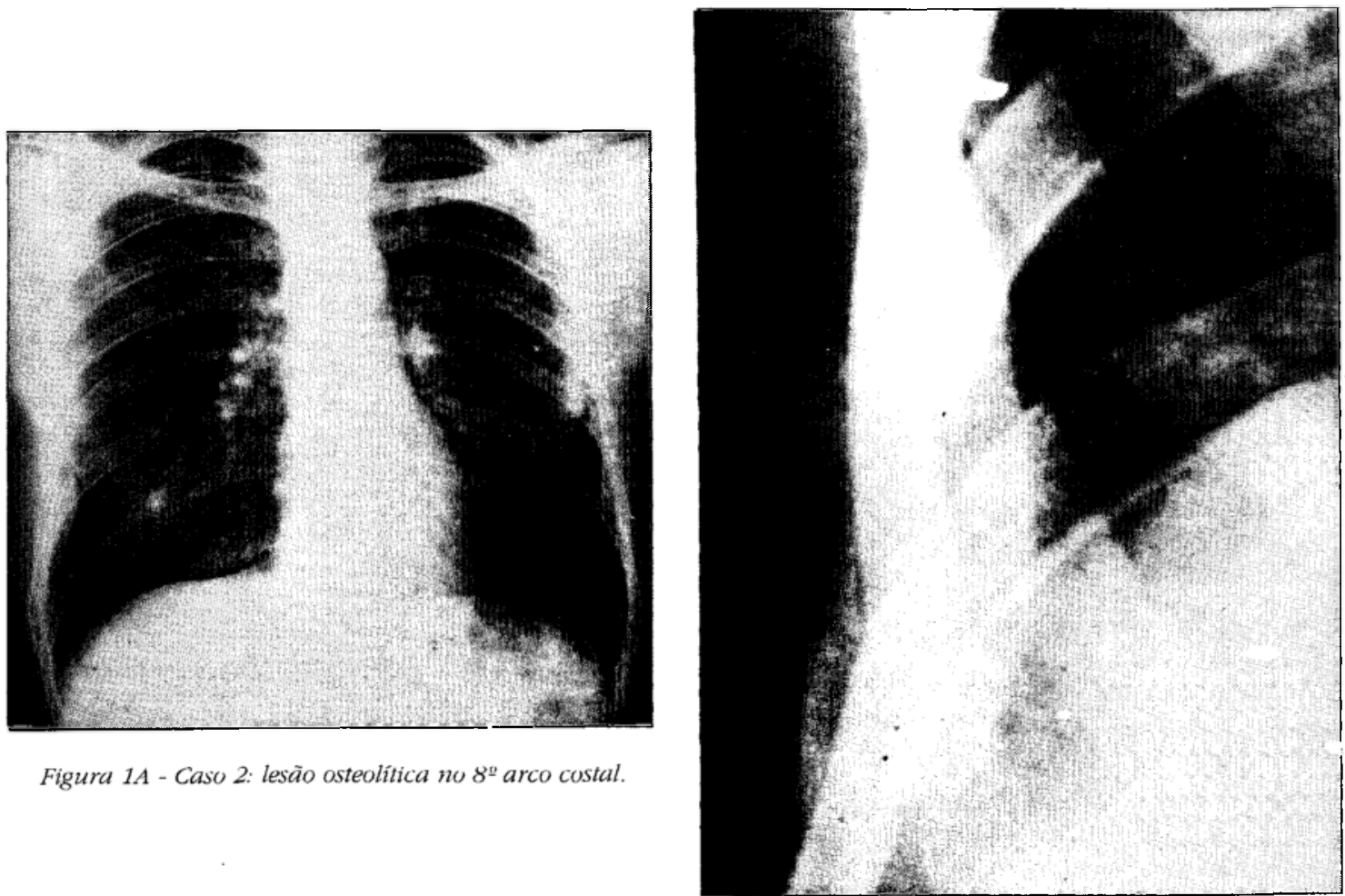

Figura $1 A$ - Caso 2: lesão osteolítica no $8^{\underline{a}}$ arco costal.

Figura 1B - Gaso 2: visão focada da lesão costal, à direita. 
Severo LC, Agostini AA, Londero AT. Acometimento ósseo na paracoccidioidonicose crônica disseminada. Relato dos primeiros casos no Rio Grande do Sul. Revista da Sociedade Brasileira de Medicina Tropical 29:241-244, mai-jun, 1996.

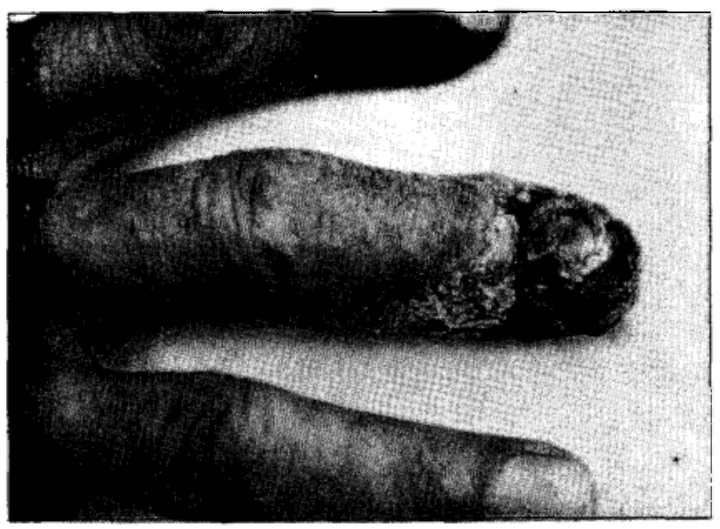

Figura 2 - Caso 3: aspecto lesional do dedo médio da máo sireita.

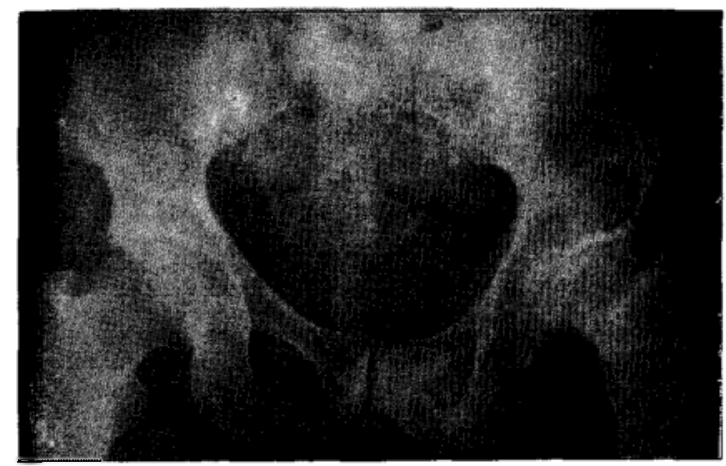

Figura í - Caso 5: lesão usteolitica do osso coxal e deformação da cabeça do fêmur à esquerda.

ou articular constitui a apresentação e/ou a manifestação mais proeminente da micose ${ }^{11}$.

No entanto, no Rio Grande do Sul ainda não havia sido feita referência ao envolvimento ósseo em doentes nativos, o que constituía, ao lado da inexistência de casos de PCM disseminada "juvenil", uma das peculiaridades da micose nesse estado. Mesmo com o relato de cinco casos, apresentados agora, continua uma curiosidade: nesses pacientes, todos adultos, apresentando a forma disseminada crônica da micose, as lesões ósseas são localizadas (solitárias) em um osso ou articulação. Nos pacientes de outros estados, as lesôes solitárias constituem minoria: 4 em 20 pacientes ${ }^{6}$, ou 3 em $15^{14}$, confira na Tabela 2. Ademais, 4 dos 5 pacientes sulinos apresentavam envolvimento pulmonar

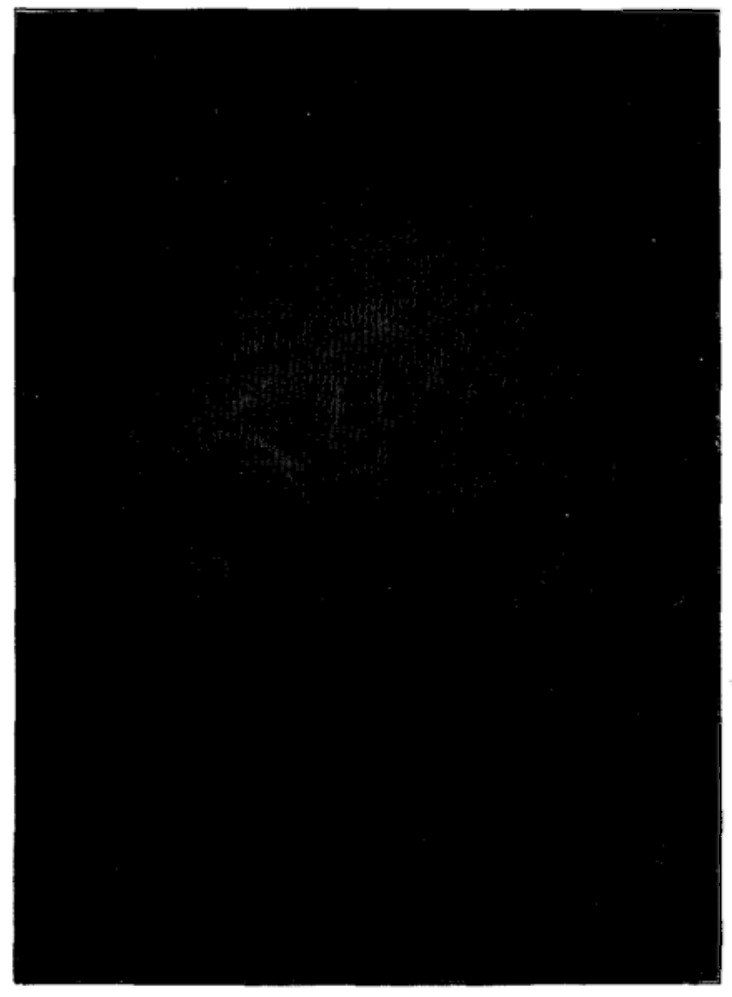

Figura 3-Caso 4: lesão usteolítica do usso frontal à direita.

concomitante, achado incomum nos pacientes do Rio de Janeiro', porém, mais comum nos doentes do Paraná ${ }^{10}$. Num dos pacientes de Silva ${ }^{10}$, curiosamente apesar do radiograma torácico não revelar anormalidade, $P$. brasiliensis era abundante no escarro.

Tabela 2 - Prevalência de lesōes osteoarticulares solitárias na PCM em casuísticas de algums estados brasileiros e em revisão da literatura.

\begin{tabular}{llrrr} 
Citercitura. & & & \\
\hline Osso ou articulação & $\mathrm{RS}^{*}$ & $\mathrm{RJ}^{6}$ & $\mathrm{PR}^{10}$ & Iiteratura \\
\hline Fêmur & $1 / 5$ & $1 / 20$ & $2 / 15$ & $1 / 92$ \\
Costela & $1 / 5$ & $1 / 20$ & $2 / 15$ & $2 / 92$ \\
Falange & $1 / 5$ & - & - & $1 / 92$ \\
Osso frontal & $1 / 5$ & $1 / 20$ & - & $5 / 92$ \\
Quadril & $1 / 5$ & $1 / 20$ & - & $2 / 92$ \\
\hline
\end{tabular}

* Presente série de casos

Numerador $=$ número de casos, Denominador $=$ número total de casos. 
Severo LC, Agostini AA, Lonḋero AT. Acometimento ósseo na paracoccidioidomicose crônica disseminada. Relato dos primeiros casos 10 Rio Grande do Sul. Revista da Sociedade Brasileira de Medicina Tropical 29:241-244, mai-jun, 1996.

Ademais, quanto ao sexo, todos os pacientes sulinos eram homens, enquanto, em outros estados a relação homem/mulher foi de $1,5: 1^{\circ}$ e $2: 1^{10}$. Um único paciente do sul apresentava sintomas de acometimento osteoarticular, porém, como usualmente ocorre, com sintomatologia inespecífica ${ }^{67} 1011$.

O diagnóstico de lesões paracoccidióidicas solitárias, desacompanhadas de outras lesões de fácil acesso, usualmente constitui achado casual em material obtido por biópsia.

\section{SUMMARY}

The first cases of bone involvement in paracoccidioidomycosis observed in the state of Rio Grande do Sul (Brazil) are reported. Clinical and radiological findings are commented and some peculiarities are pointed out.

Key-words: Paracoccidioidomycosis Paracoccidioides brasiliensis. Bone. Joint.

\section{REFERÊNCIAS BIBLIOGRÁFICAS}

1. Barbosa GL. Paracoccidioidomicose na criança. Tese de Mestrado, Instituto de Patologia Tropical, Universidade Federal de Goiás, Goiânia, 1992.

2. Barbosa W. Blastomicose sul-americana. Contribuição ao seu estudo no Estado de Goiás. Tese de Livre-Docência, Faculdade de Medicina, Universidade Federal de Goiás, Goiânia, 1968.

3. Campos EC. Micose de Lutz (Blastomicose sulamericana). Contribuição ao seu estudo no Estado do Rio Grande do Sul. Tese de LivreDocência, Faculdade de Medicina, Universidade Federal do Rio Grande do Sul, Porto Alegre, 1960.

4. Londero AT, Ramos CD. Paracoccidioidomicose. Estudo clínico e micológico de 260 casos observados no interior do Estado do Rio Grande do Sul. Journal de Pneumologia 16: 129-132, 1990.

5. Machado Filho J, Miranda JL. Considerações relativas à blastomicose sul-americana. Localizações, sintomas iniciais, vias de penetração e disseminação em 313 casos consecutivos. $O$ Hospital 58: 99-137, 1960.

6. Marchiori E. Aspectos radiológicos das lesões osteoarticulares na paracoccidiodomicose considerações sobre 20 casos. Radiologia Brasileira 22: 5-16, 1989.

7. Nanni L. Aspectos radiológicos da paracoccidiodomicose óssea. Tese de Doutodo, Faculdade de Ciências Médicas, Universidade Estadual de Campinas, Campinas, 1976.

8. Pereira M,Vianna GO. A propósito de um caso de blastomycose (Piohemnia blastomycotica) Archivo Brasileiro de Medicina 1: 63-83, 1911.

9. Severo LC, Geyer GR, Londero AT, Porto NS, Rizzon CFC. Primary lymph node complex in paracoccidioidomycosis. Mycopathologia 67: 115-118, 1979.

10.Silva AF. Contribuição aos estudos da paracoccidioidomicose no Paraná e das lesões ósseas e articulares paracoccidióidicas. Tese de Livre-Docência, Curso de Medicina, Universidade Federal do Paraná, Curitiba, 1976.

11. Terra GMF. Contribuição ao estudo clínico da paracoccidioidomicose na criança. Tese de Mestrado, Faculdade de Medicina, Universidade Federal do Rio de Janeiro, Rio de Janeiro, 1993.

12. Versiani $O$. Blastomicose. Revista Brasileira de Biologia 5: 37-60, 1945. 